\title{
COMPUTATIONS OF INVISCID COMPRESSIBLE FLOWS USING FLUCTUATION-SPLITTING ON TRIANGULAR MESHES
}

\author{
H. Paillère* - H. Deconinck* - R. Struijs* \\ P.L. Roe $\nmid$ - L.M. Mesaros $\dagger$ - J.-D. Müller*† \\ * von Karman Institute for Fluid Dynamics, Belgium \\ $\dagger$ The University of Michigan, Ann Arbor, USA
}

\begin{abstract}
The multidimensional upwind approach for the Euler equations discussed in this paper generalizes to $2 \mathrm{D}$ the wellknown flux difference scheme of Roe. The method, which uses grids composed of triangles, is based on a conservative decomposition of the flux balance for each cell into scalar wave contributions, which are then upwinded to the vertices using a high-resolution compact monotone scalar advection scheme. Whereas the advection part and the conservative linearization have been extensively treated in the past, the present paper concentrates on the choice of the wave model generalizing the characteristic decomposition at the base of the $1 \mathrm{D}$ flux difference splitters. Contrary to the $1 \mathrm{D}$ case, many possibilities exist and a thorough review and comparison of existing and new models are given, emphasizing performance in subsonic as well as supersonic flows. The numerical results presented for a wide range of internal and external flows show the strong potential of the method.
\end{abstract}

\section{Introduction}

In the last fifteen years, upwind finite volume methods have emerged as a robust and rather accurate tool in computing multidimensional compressible flows. Nevertheless, most of these schemes rely on $1 \mathrm{D}$ physics, i.e the Riemann problem that describes the interaction between two fluid states separated by a cell face, by waves propagating in the direction of its normal. Typically, this leads to a misinterpretation and unwanted diffusion of shocks or shear layers not aligned with the grid. To cope with this basic weakness, multidimensional elements have been incorporated in the interface fluxes, to allow waves to propagate in more physical directions $[1,2]$.

While these attempts have met with some degree of success (see the review papers by Roe and van Leer $[3,4]$ ), the present approach starts from a totally different viewpoint: instead of trying to cure the interface fluxes in a finite volume approach, we start from a different interpretation of Roe's flux difference scheme in 1D, namely as a cell vertex residual distribution scheme.
A multidimensional generalization then leads to a fluxbalance splitting scheme over triangular cells in $2 \mathrm{D}$ and tetrahedral cells in 3D. Thus, to construct a method based on this idea, three basic ingredients are needed:

- an analytical model to decompose the $2 \mathrm{D}$ or $3 \mathrm{D}$ flux divergence into scalar wave contributions;

- a discrete version which preserves conservation.

- a cell vertex scheme for the scalar advection equation which governs each scalar wave,

$$
\frac{\partial W}{\partial t}+\vec{\lambda} \cdot \nabla W=0
$$

Each of these ingredients has evolved substantially over the last years and all three have reached a degree of maturity which almost allows routine computation of standard 2D inviscid flows, as is shown in this paper.

Concerning the advection step, Roe [5] presented the first fluctuation-splitting schemes on triangles in 1987. Soon after, Struijs et al. [6] proposed a number of non-linear schemes satisfying crucial properties such as positivity and linearity preservation. Extension to 3D and further refinements were made in 1992 by Bourgois et al. [7]. Paillère [8] and Mesaros (unpublished results) made a study of the shock capturing of the non-linear scalar advection schemes for particular grid configurations, thus greatly improving their understanding. An exhaustive discussion of the advection schemes is given in [9], including a detailed accuracy study.

The generalization of the 1D Roe linearization to triangles and tetrahedra turned out to be very simple and was discovered independently by Roe and Struijs $[10,11]$ in 1991.

While the scalar advection step and the conservative linearization are now fairly well understood, the wave modeling step is still a topic of further study, and the major purpose of this paper is to discuss the many recent developments in this area. Indeed, contrary to the ID case where the wave decomposition of the residual is unique, waves in $2 \mathrm{D}$ can travel in an infinite number 
of directions ; to define the most physically relevant directions and the number of active waves is the task of the wave model. The first developments in multidimensional wave modeling date from 1986, with the simple wave models of Roe [12] and the characteristic decomposition of Deconinck et al. [13]. Other important contributions were made in the following years (De Palma et al. [14], Roe and Mesaros [15]). In 1992 (see [16] for a detailed account), Rudgyard [17] proposed a new family of models while Parpia [18] proposed a new wave model applied in the context of finite volume methods. Very recently, Roe developed yet another model which incorporates both steady patterns (which do not contribute to the residual) and unsteady simple waves [19]. A thorough evaluation and comparison of these wave models is made in the present paper, including the first results for Parpia's model and for the latest Roe models.

The paper is organized as follows: in sections 2 and 3, the scalar advection schemes and the conservative linearization are briefly recalled. The different wave models are described in section 4. A detailed analysis of the performance of the solver in subsonic flow is made in section 5 , highlighting some severe deficiencies of existing models as well as the improvements brought by the latest model. In section 6 , the accuracy of the solver is evaluated, which confirms the analysis performed in the scalar case, where the monotone non-linear scheme was found to be of order 1.6. Finally, section 7 gives an overview of the performance of the method for a number of standard testcases.

\section{The scalar advection equation}

Fluctuation-splitting is a cell-vertex space discretization for the scalar advection equation,

$$
\frac{\partial W}{\partial t}+\vec{\lambda} \cdot \nabla W=0
$$

where $\vec{\lambda}$ is the advection vector, which depends on the space coordinates and eventually on the solution itself. For simplicity, let it be a constant vector. Assuming a continuous piecewise linear representation of $W$ on a triangular mesh, the residual or "fluctuation" for each triangle with surface $S_{T}$ can be computed as:

$$
\phi_{T}=\iint_{T} W_{t} d \Omega=-S_{T} \vec{\lambda} \cdot \nabla W=-\sum_{i=1}^{3} W_{i} k_{i},
$$

where $k_{i}=\frac{1}{2} \vec{\lambda} \cdot \vec{n}_{i}$ and $\vec{n}_{i}$ are the inward scaled normals of the triangle, see fig 1 . Considering simple Euler explicit time integration, all compact distribution schemes can be cast into the following forms, for mesh point $i$ :

$$
\begin{aligned}
W_{i}^{n+1} & =W_{i}^{n}+\frac{\Delta t}{S_{i}} \sum_{T} \beta_{T, i} \phi_{T} \\
& =W_{i}^{n}+\frac{\Delta t}{S_{i}} \sum_{T} \gamma_{T, i},
\end{aligned}
$$

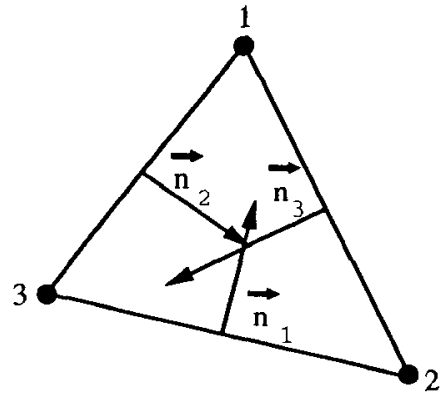

Figure 1: cell-vertex triangle

where the summation extends over all triangles having $i$ as common vertex. Further, for conservation and consistency, the coefficients $\beta_{T, j}$ and the functions $\gamma_{T, j}$ are chosen such that for each individual triangle $T$ with vertices $j, \sum_{j=1}^{3} \beta_{T, j}=1$ and $\sum_{j=1}^{3} \gamma_{T, j}=\phi_{T}$. For compactness of the stencil, the distribution is done on a triangle basis, and only involves the nodes of each triangle. $S_{i}$ is the area of the median dual cell around node $i$. For an upwind scheme, the weights $\beta_{T, i}$ depend on the advection speed $\vec{\lambda}$ : only downwind nodes receive a contribution. This can be expressed as:

$$
\gamma_{T, i}=\beta_{T, i}=0 \quad \text { if } \quad k_{i}<0 .
$$

Both positive and linearity-preserving schemes have been developed. To combine both properties, the schemes have to be made non-linear, i.e the $\gamma_{T, i}$ have to be non-linear functions of the data. In this paper, three particular schemes have been used: the linear $\mathrm{N}$ and LDA (Low Diffusion A) schemes, and the non-linear PSI (Positive Streamwise Invariant) scheme. Compact formulae have been derived for each of these schemes [7]:

$$
\begin{aligned}
\gamma_{T, i}^{N} & =-\max \left(0, k_{i}\right)\left(W_{i}-W_{i n}\right) \\
\gamma_{T, i}^{L D A} & =\frac{\max \left(0, k_{i}\right)}{\sum_{j=1}^{3} \max \left(0, k_{j}\right)} \phi_{T} \\
\gamma_{T, i}^{P S I} & =\frac{\max \left(0, k_{i}\right) \min \left(0,\left(W_{i}-W_{i n}\right) \phi_{T}\right)}{\sum_{j=1}^{3} \max \left(0, k_{j}\right) \min \left(0,\left(W_{j}-W_{i n}\right) \phi_{T}\right)} \phi_{T}
\end{aligned}
$$

where $W_{i n}$ is the interpolated solution at the inflow point of the triangle (fig 2) given by:

$$
W_{i n}=\frac{\sum_{j=1}^{3} W_{j} \min \left(0, k_{j}\right)}{\sum_{j=1}^{3} \min \left(0, k_{j}\right)} .
$$

These schemes are all upwind in the sense that no contribution is sent to upstream vertices. Fig 2 shows the two possible situations that can occur in a triangle. In $2 \mathrm{a}$ ), the fluctuation is split between nodes 1 and 2 $\left(k_{1}>0, k_{2}>0, k_{3}<0\right)$, and in $\left.2 \mathrm{~b}\right)$ the whole fluctuation is sent to node $2\left(k_{2}>0, k_{1}<0, k_{3}<0\right)$. From the formulae given above, it is clear that the $\mathrm{N}$ and LDA schemes are linear, whereas the PSI is not, and 


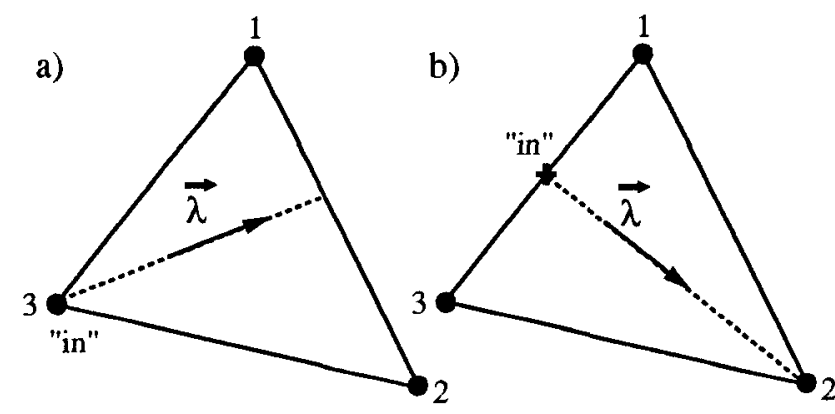

Figure 2: a) two-target situation b) one-target situation

that the LDA and PSI schemes preserve linear steadystate solutions $\left(\gamma_{T, i} \rightarrow 0\right.$ when $\left.\phi_{T} \rightarrow 0\right)$ unlike the $\mathrm{N}$ scheme. Furthermore, the $\mathrm{N}$ and PSI schemes can be made positive under a CFL condition, unlike the LDA scheme. The three schemes are also continuous for changes of advection speed $\vec{\lambda}$ as well as for changes in the solution, unlike the non-linear NN scheme used previously [20]. Continuity is a requirement for robustness of the schemes, since it allows smooth transitions of the solution, and so avoids limit cycle behaviour in the convergence. In all scalar cases run, convergence to machine zero is obtained each time for all of the three schemes considered.

In [9], an accuracy study was also made to estimate the order of the schemes for smooth solutions. Using the $L_{2}$ norm, the $\mathrm{N}$ scheme was found to be of order 0.85 , the PSI 1.6 and the LDA exactly 2.0 .

\section{The Euler equations}

The Euler equations in conservative form are:

$$
\mathrm{U}_{t}+\mathbf{F}_{x}+\mathbf{G}_{y}=\mathbf{0}
$$

where $\mathbf{U}$ is the vector of conserved variables and $\mathbf{F}$ and $\mathbf{G}$ are the flux vectors:

$$
\mathbf{U}=\left(\begin{array}{c}
\rho \\
\rho u \\
\rho v \\
\rho E
\end{array}\right) \mathbf{F}=\left(\begin{array}{c}
\rho u \\
\rho u^{2}+p \\
\rho u v \\
\rho u H
\end{array}\right) \mathbf{G}=\left(\begin{array}{c}
\rho v \\
\rho u v \\
\rho v^{2}+p \\
\rho v H
\end{array}\right) .
$$

For a system of equations, the fluctuation-splitting schemes are based on a "wave" decomposition of the residual in each cell. This wave modeling step is discussed in detail in the next section. Very generally, the flux divergence can be written as

$$
\mathbf{U}_{t}=-\mathbf{F}_{x}-\mathbf{G}_{y}=-\sum_{k}\left(\nabla W^{k} \cdot \vec{\lambda}^{k}\right) \mathbf{R}^{k}
$$

where $W^{k}$ is a scalar, and $\mathbf{R}^{k}$ its projection onto the conservative variables. Thus, the solution evolves in time according to a set of scalar equations:

$$
W_{t}^{k}+\vec{\lambda}^{k} \cdot \nabla W^{k}=0
$$

by projection onto the vectors $\mathbf{R}^{k}$. To construct a conservative scheme for the Euler equations based on wave-decomposition and upwind distribution, a particular linearization is applied [10]. As in the scalar case, cell-vertex triangular meshes are considered: at each time-step, and in each cell, the state vector $\mathbf{Z}=(\sqrt{\rho}, \sqrt{\rho} u, \sqrt{\rho} v, \sqrt{\rho} H)^{\tau}$ is assumed to vary linearly. This vector is such that $\mathbf{U}, \mathbf{F}$ and $\mathbf{G}$ are quadratic in its components, leading to an easy linearization of the flux balance in each cell:

$$
\oint_{\partial T}\left(\mathbf{F} n_{x}+\mathbf{G} n_{y}\right) d l=S_{T} \sum_{k}\left(\nabla W^{k} \cdot \widehat{\vec{\lambda} k}\right) \widehat{\mathbf{R}}^{k}
$$

where $S_{T}$ is the area of the cell and the hat $\widehat{()}$ denotes the evaluation at the average state of the cell,

$$
\widehat{\mathbf{Z}}=\frac{\mathbf{Z}_{1}+\mathbf{Z}_{2}+\mathbf{Z}_{3}}{3}
$$

Applying now the scalar advection scheme to each wave, the residual of the cell can be distributed in a conservative way to the nodes. After performing the residual distribution step, the solution can be updated. At present, explicit time-stepping is used, with Forward Euler or Runge-Kutta type schemes. The final update formula can be written as

$$
\mathbf{U}_{i}^{n+1}=\mathbf{U}_{i}^{n}-\frac{\Delta t}{S_{i}} \sum_{T} \sum_{k} \beta_{T, i}^{k} S_{T}\left(\nabla W^{k} \cdot \widehat{\overrightarrow{\lambda^{k}}}\right) \widehat{\mathbf{R}}^{k}
$$

where the summation index $T$ carries over all triangles having $i$ as a common vertex, while $\beta_{T, i}^{k}$ represents the fraction of the residual of the $k^{t h}$ wave in cell $T$ sent to node $i$.

At the boundaries, two approaches can be applied: either a strong imposition using characteristic boundary conditions, or a weak form that enforces the conditions on the fluxes. The latter allows the use of interior schemes at the boundary and can enhance the robustness of the solver.

\section{Wave models}

In one dimension, the solution can be projected uniquely onto the eigenvectors of the flux Jacobian, leading to the decomposition of the flux difference $\mathbf{F}_{x}$ into waves or "characteristics" traveling along the axis:

$$
\mathbf{U}_{x}=\sum_{k} W_{x}^{k} \mathbf{R}^{k}, \quad \mathbf{F}_{x}=\sum_{k=1}^{3} \lambda^{k} W_{x}^{k} \mathbf{R}^{k}
$$

In $2 \mathrm{D}$, the decomposition of the flux divergence into scalar waves is no longer unique. At present, three different approaches exist and are compared in this paper. The first one is based on characteristic compatibility equations of the Euler system, the second one on simple 
wave decompositions of the solution, and the third one on a projection of the solution onto a basis of steady and unsteady patterns. Each of these families allows many variants. We begin with a brief presentation of all the models.

\subsection{Characteristic decomposition}

This decomposition [13] is based on a particular choice of a vector of characteristic variables $\partial \mathbf{W}\left(\vec{\kappa}^{(1)}, \vec{\kappa}^{(2)}\right)$, which allows an optimum decoupling of the Euler system for certain choices of $\vec{\kappa}^{(1)}$ and $\vec{\kappa}^{(2)}$. The characteristic variables $\partial \mathbf{W}$ are given by

$$
\partial \mathbf{W}=\left(\begin{array}{c}
\partial \rho-\partial p / a^{2} \\
\vec{s}^{(1)} \cdot \partial \vec{u} \\
\frac{1}{2}\left(\vec{\kappa}^{(2)} \cdot \partial \vec{u}+\partial p / \rho a\right) \\
\frac{1}{2}\left(-\vec{\kappa}^{(2)} \cdot \partial \vec{u}+\partial p / \rho a\right)
\end{array}\right)=\mathbf{P}^{*-1} \partial \mathbf{U}
$$

where $\mathbf{P}^{*-1}$ is the transformation matrix from conservative to characteristic variables. Thus $\partial \mathbf{U}=\mathbf{P}^{*} \partial \mathbf{W}$. $\vec{s}^{(1)}$ is the vector perpendicular to $\vec{\kappa}^{(1)}$. Substituting into the Euler system, the following system of compatibility equations is obtained:

$$
\begin{aligned}
W_{t}^{1}+\vec{u} \cdot \nabla W^{1}+q^{1} & =0 \\
W_{t}^{2}+\vec{u} \cdot \nabla W^{2}+q^{2} & =0 \\
W_{t}^{3}+\left(\vec{u}+a \vec{\kappa}^{(2)}\right) \cdot \nabla W^{3}+q^{3} & =0 \\
W_{t}^{4}+\left(\vec{u}-a \vec{\kappa}^{(2)}\right) \cdot \nabla W^{4}+q^{4} & =0
\end{aligned}
$$

where the $q^{k}$ are coupling terms:

$q^{1}=0, q^{2}=a s^{(1)} \cdot\left(\nabla W^{3}+\nabla W^{4}\right)=s^{(1)} \cdot \nabla p / \rho$ and $q^{3}=q^{4}=f\left(\nabla u, \nabla v, \vec{\kappa}^{(1)}, \vec{\kappa}^{(2)}\right) . q^{2}$ can be set to zero by choosing $\vec{\kappa}^{(1)}$ parallel to the pressure gradient, and $q^{3}=q^{4}$ can be minimized for certain choices of $\vec{\kappa}^{(2)}$. Finally, the cell residual is decomposed as:

$$
\begin{aligned}
\Phi & =-S_{T} \sum_{k=1}^{4}\left(\nabla W^{k} \cdot \widehat{\vec{\lambda}^{k}}+q^{k}\right) \widehat{\mathbf{R}}^{k} \\
& =-S_{T} \sum_{k=1}^{4}\left(\nabla W^{k} \cdot \widehat{\vec{\lambda}^{k}}\right){\widehat{\mathbf{R}^{*}}}^{k}-S_{T} \widehat{\mathbf{Q}}
\end{aligned}
$$

with $\vec{\lambda}^{1}=\vec{\lambda}^{2}=\vec{u}, \vec{\lambda}^{3,4}=\vec{u} \pm a \vec{\kappa}^{(2)}$, while $\widehat{\mathbf{R}}^{k}$ are the columns of the matrix $\widehat{\mathbf{P}}^{*}$, and $\widehat{\mathbf{Q}}$ is the coupling term expressed in conservative variables. To distribute the coupling term, two possible alternatives exist: either to equidistribute $\widehat{\mathbf{Q}}$ to the three nodes (eq (12)), or to upwind the $q^{k}$ in the same way as the advective part (eq (11)).

\subsection{Simple wave decompositions}

This decomposition method relies on a "pattern recognition" step, by which the local flow gradients are modeled by a set of simple waves. Simple waves are elementary solutions of the Euler equations for linearized flow, of the form

$$
\mathbf{U}(\vec{x}, t)=\alpha\left(\vec{x} \cdot \vec{m}-\lambda_{m} t\right) \mathbf{R}_{m}+\mathbf{U}_{0}
$$

where $\alpha$ and $\vec{m}=\cos \theta \overrightarrow{1}_{x}+\sin \theta \overrightarrow{1}_{y}$ are constants representing respectively the wave strength and the direction of propagation of the wave front, and $\lambda_{m}$ and $\mathbf{R}_{m}$ are corresponding eigenvalue and eigenvector of the matrix

$$
\frac{\partial \mathbf{F}}{\partial \mathbf{U}} \cos \theta+\frac{\partial \mathbf{G}}{\partial \mathbf{U}} \sin \theta \text {. }
$$

The eigenvalues, $\lambda_{m}^{a \pm}=\vec{u} \cdot \vec{m} \pm a$ and $\lambda_{m}^{s, e}=\vec{u} \cdot \vec{m}$, are the wave speeds corresponding respectively to two acoustic waves, a shear wave and an entropy wave.

The requirement for a simple wave model is that it has enough degrees of freedom to match any arbitrary linear variation of the data. Thus in 2D, a model should have 8 degrees of freedom, represented by the unknowns $\alpha^{k}$ and $\theta^{k}$. In practice, these are derived from the decomposition of the linear variations of the primitive variables $\mathbf{V}=(\rho, u, v, p)^{\tau}$ :

$$
\nabla \mathbf{V}=\sum_{k} \alpha^{k} \mathbf{P}_{u}^{k}
$$

where $\mathbf{P}_{u}^{k}$ is the $2 \times 4$ matrix or pattern describing the linear flow variations due to the unsteady simple wave $k$. For instance, the patterns for an entropy wave and a shear wave propagating in the directions $\theta^{e}$ and $\theta^{s}$ are respectively:

$$
\begin{aligned}
& \mathbf{P}_{u}^{e}\left(\theta^{e}\right)=\left[\begin{array}{cc}
\cos \theta^{e} & \sin \theta^{e} \\
0 & 0 \\
0 & 0 \\
0 & 0
\end{array}\right] \\
& \mathbf{P}_{u}^{s}\left(\theta^{s}\right)=\left[\begin{array}{cc}
0 & 0 \\
-\sin \theta^{s} \cos \theta^{s} & -\sin ^{2} \theta^{s} \\
\cos ^{2} \theta^{s} & \cos \theta^{s} \sin \theta^{s} \\
0 & 0
\end{array}\right],
\end{aligned}
$$

and for an acoustic propagating in the direction $\theta^{a}$,

$$
\mathbf{P}_{u}^{a}\left(\theta^{a}\right)=\left[\begin{array}{cc}
\rho \cos \theta^{a} / a & \rho \sin \theta^{a} / a \\
\cos ^{2} \theta^{a} & \cos \theta^{a} \sin \theta^{a} \\
\sin \theta^{a} \cos \theta^{a} & \sin ^{2} \theta^{a} \\
\rho a \cos \theta^{a} & \rho a \sin \theta^{a}
\end{array}\right] .
$$

Different models have been proposed following this approach: Roe's family of 6-wave models, Parpia's 5-wave model, and the "directional splitting" models of Rudgyard:

- In [12], Roe showed that a simple wave model could be produced by considering four acoustic waves propagating at $90^{\circ}$ to one another, an entropy wave and a shear wave, the latter propagating in a well-chosen direction. The acoustic waves represent 5 degrees of freedom (strengths and direction), the entropy wave 2 (strength 


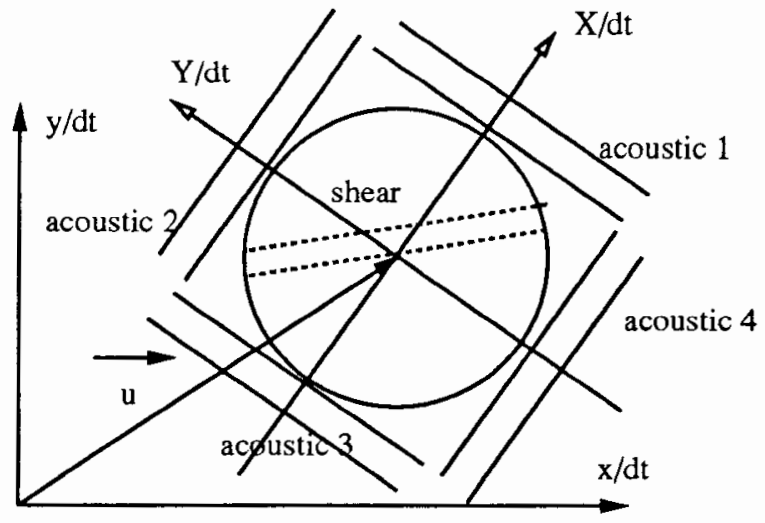

Figure 3: wave directions: model D (entropy wave not represented)

and direction) and the shear wave 1 (strength). Since the direction of the latter is free, a one-parameter family of models is obtained: Roe's original model B [12] (direction perpendicular to the velocity), model $\mathrm{C}$ of De Palma et al. [14] (direction of the pressure gradient), Roe's model D [15] (bisector of the principle axes of the strain rate tensor, $X$ and $Y$, see fig 3 ).

- Another simple wave model was developed by Parpia [18], combining two opposite acoustic waves (3 degrees: two strengths and one direction), two perpendicular shear waves (3 degrees: two strengths and one direction) and an entropy wave (2 degrees: strength and direction), see fig 4 . The directions and strengths of the five waves are:

$$
\begin{aligned}
\theta^{a} & =\arctan \frac{p_{y}}{p_{x}} \\
\alpha^{a \pm} & =\frac{1}{2}\left[u_{x}+v_{y} \pm \frac{1}{\rho a} \sqrt{p_{x}^{2}+p_{y}^{2}}\right]
\end{aligned}
$$

for the acoustic waves,

$$
\begin{aligned}
\theta^{s} & =\frac{1}{2} \arctan \frac{\left(u_{x}+v_{y}\right) \cos 2 \theta^{a}-\left(u_{x}-v_{y}\right)}{\left(v_{x}+u_{y}\right)-\left(u_{x}+v_{y}\right) \sin 2 \theta^{a}} \\
R & =\frac{\left(u_{x}+v_{y}\right) \cos 2 \theta^{a}-\left(u_{x}-v_{y}\right)}{\sin 2 \theta^{s}} \\
& =\frac{\left(v_{x}+u_{y}\right)-\left(u_{x}+v_{y}\right) \sin 2 \theta^{a}}{\cos 2 \theta^{s}} \\
\alpha^{s \pm} & =\frac{1}{2}\left[v_{x}-u_{y} \pm R\right]
\end{aligned}
$$

for the shear waves and

$$
\begin{aligned}
\theta^{e} & =\arctan \frac{\rho_{y}-\frac{p_{y}}{a^{2}}}{\rho_{x}-\frac{p_{x}}{a^{2}}} \\
\alpha^{e} & =\sqrt{\left(\rho_{x}-\frac{p_{x}}{a^{2}}\right)^{2}+\left(\rho_{y}-\frac{p_{y}}{a^{2}}\right)^{2}}
\end{aligned}
$$

for the entropy wave.

- Another family of models was recently developed by

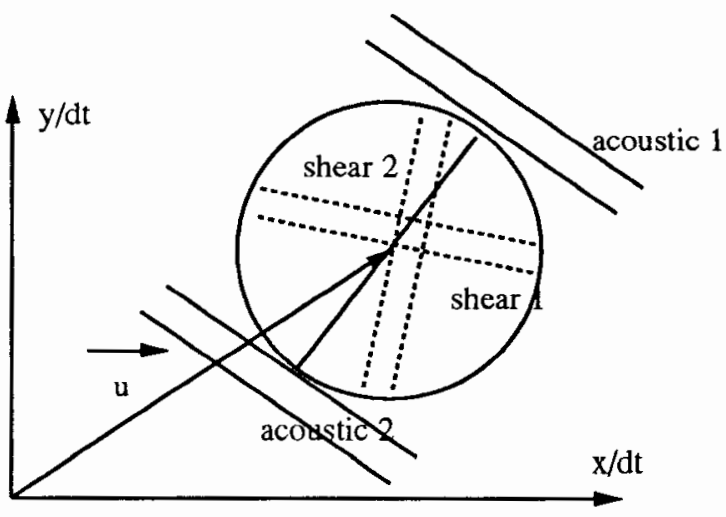

Figure 4: wave directions: Parpia's model (entropy wave not represented)

Rudgyard [16]. The starting point is the decomposition of $\nabla \mathbf{V}$ into an entropy wave pattern, and two sets of patterns (each one comprises two acoustic waves and a shear wave) depending on two arbitrary directions $\theta_{1}$ and $\theta_{2}$ :

$$
\nabla V=\sum_{k=1}^{3} \alpha_{1}^{k} \mathbf{P}_{u}^{k}\left(\theta_{1}\right)+\sum_{k=1}^{3} \alpha_{2}^{k} \mathbf{P}_{u}^{k}\left(\theta_{2}\right)+\alpha_{e} \mathbf{P}_{u}^{e}
$$

The model is complete since there are 8 unknowns, the strength and direction of the entropy wave, and the strengths of the 4 acoustic and 2 shear waves. By choosing the angles $\theta_{1}$ and $\theta_{2}$, a two-parameter family of models is obtained, consisting of 7 waves. This is clearly computationally expensive, and can lead to unnecessary dissipation, as will be shown later. Rudgyard therefore proposed to reduce the number of active waves by choosing particular directions for which some of the wave speeds are zero. For instance, taking the directions parallel and perpendicular to the velocity yields a 6 -wave model called the "streamwise splitting". A less diffusive model, called the "Mach angle splitting" and valid for supersonic flow only, is obtained by taking the directions normal to the Mach lines, see fig 5 :

$$
\tan \theta_{1}=\frac{u \sqrt{M^{2}-1}+v}{u-v \sqrt{M^{2}-1}} \quad \tan \theta_{2}=\frac{v-u \sqrt{M^{2}-1}}{u+v \sqrt{M^{2}-1}}
$$

Finally, for all these models, the cell residual can be written as:

$$
\Phi_{T}=-S_{T} \sum_{k}\left(\nabla W^{k} \cdot \widehat{\vec{\lambda} k}\right) \widehat{\mathbf{R}}_{m}^{k}
$$

where we define $\nabla W^{k}=\alpha^{k} \vec{m}^{k}, \vec{\lambda}^{k}=\vec{u}$ for the shear and entropy wave, and $\vec{\lambda}^{k}=\vec{u}+a \vec{m}^{k}$ for the acoustic waves. The wave speed is then given by $\lambda_{m}^{k}=\vec{\lambda}^{k} \cdot \vec{m}^{k}$. However, as pointed out by Rudgyard [16], there is no 


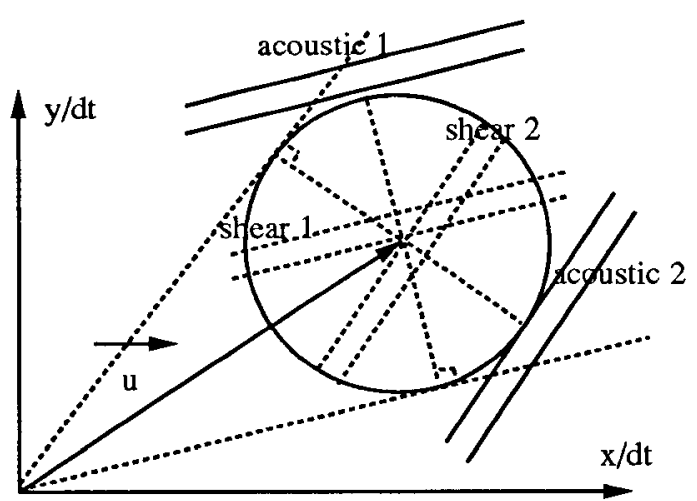

Figure 5: wave directions: Mach angle splitting (entropy wave not represented)

clear justification for this choice: we cannot assume in a decomposition involving more than one simple wave, that those waves do indeed propagate in the directions $\vec{m}^{k}$. Using a different approach than was presented here, he proposed other expressions for $\nabla W^{k}$ and $\vec{\lambda}^{k}$, leading to a different implementation.

\subsection{Steady pattern decomposition}

At present, neither the characteristic approach nor the simple wave models as presented above have proven entirely satisfying, in particular when computing subsonic flows. It was observed that the fewer waves used, the more accurate the solution. This observation confirms the idea that using more waves than there are unknowns (as occurs in all the above models except for the characteristic model) leads to unnecessary numerical dissipation, as will be shown later. Recently [19], Roe proposed to match the local data gradient with a combination of steady patterns (which do not contribute to the residual) and unsteady simple waves. Thus one may hope that at steady state, all unsteady waves would vanish, i.e $\alpha^{k} \lambda_{m}^{k} \rightarrow 0 \forall k$, and the solution could be interpreted as the superposition of steady patterns rather than the cancellation of unsteady simple waves.

Starting from the quasilinear form of the Euler equations,

$$
\mathbf{V}_{t}+\mathbf{A V}_{x}+\mathbf{B V}_{y}=\mathbf{0}
$$

a basis of linearly independent steady state solutions can be constructed by considering a steady entropy layer, a steady shear layer and two potential (isentropic, irrotational) flow solutions:

$$
\mathbf{P}_{s}^{e}=\left[\begin{array}{cc}
v & -u \\
0 & 0 \\
0 & 0 \\
0 & 0
\end{array}\right]
$$

$$
\begin{aligned}
\mathbf{P}_{s}^{s}= & {\left[\begin{array}{cc}
0 & 0 \\
-u v & u^{2} \\
-v^{2} & u v \\
0 & 0
\end{array}\right] } \\
\mathbf{P}_{s}^{p o t 1}= & {\left[\begin{array}{cc}
\rho v & -\rho u \\
-2 u v / M^{2} & \left(u^{2}-v^{2}\right) / M^{2} \\
\left(u^{2}-v^{2}\right) / M^{2} & 2 u v / M^{2} \\
\rho a^{2} v & -\rho a^{2} u
\end{array}\right] } \\
\mathbf{P}_{s}^{p o t 2}= & \left.\begin{array}{cc}
\rho u & \rho v \\
-\left(u^{2}+\eta v^{2}\right) / M^{2} & u v(\eta-1) / M^{2} \\
u v(\eta-1) / M^{2} & -\left(v^{2}+\eta u^{2}\right) / M^{2} \\
\rho a^{2} u & \rho a^{2} v
\end{array}\right],
\end{aligned}
$$

where $\eta=M^{2}-1$ and $M$ is the Mach number. Note that in the first potential solution pattern, the pressure gradient is normal to the flow, and in the second, it is aligned with it. By definition, the $\mathbf{P}_{s}^{i}$ satisfy

$$
(\mathbf{A}, \mathbf{B}) \cdot \mathbf{P}_{s}^{i}=\mathbf{0} \text {. }
$$

A model combining both steady patterns $\mathbf{P}_{s}$ and unsteady simple waves $\mathbf{P}_{u}$ is now considered:

$$
\nabla \mathbf{V}=\sum_{i} \beta^{i} \mathbf{P}_{s}^{i}+\sum_{k} \alpha^{k} \mathbf{P}_{u}^{k}
$$

Just as before, this model should have 8 degrees of freedom, represented by the $\beta^{i}, \alpha^{k}$ and $\theta^{k}$. Roe suggested taking the steady patterns corresponding to the shear and potential flow solutions ( 3 degrees), as well as an unsteady entropy wave (2 degrees) and two acoustic waves propagating in opposite directions (3 degrees).

As in all previous models, expressions for the strength and direction of the entropy wave are given by the norm and direction of the entropy gradient. For the acoustic waves, the algebra is much more difficult. However, taking the product of $\nabla \mathbf{V}$ with $(\mathbf{A}, \mathbf{B})$ eliminates all mention of the steady terms. Finally, extremely simple expressions are obtained:

$$
\begin{aligned}
\theta & =\arctan \frac{v_{t}}{u_{t}} \\
\alpha^{+} \lambda_{m}^{+} & =\frac{1}{2}\left[\sqrt{u_{t}^{2}+v_{t}^{2}}-p_{t} / \rho a\right] \\
\alpha^{-} \lambda_{m}^{-} & =\frac{1}{2}\left[\sqrt{u_{t}^{2}+v_{t}^{2}}+p_{t} / \rho a\right],
\end{aligned}
$$

with $\lambda_{m}^{ \pm}=u \cos \theta+v \sin \theta \pm a$. In this model, the residual reduces to a 3 -wave decomposition:

$$
\Phi_{T}=-S_{T} \sum_{k} \alpha^{k} \lambda_{m}^{k} \widehat{\mathbf{R}}_{m}^{k}
$$

Expressions for the intensities of the steady patterns, which are not actually needed since only unsteady terms appear in the residual, are given by:

$$
\beta^{s}=-\frac{v_{x}-u_{y}}{u^{2}+v^{2}}
$$




$$
\begin{aligned}
\beta^{\text {pot } 1} & =\frac{v p_{x}-u p_{y}-\left(\alpha^{+}-\alpha^{-}\right) \rho a u_{\|}}{\rho a^{2}\left(u^{2}+v^{2}\right)} \\
\beta^{\text {pot2 }} & =\frac{u p_{x}+v p_{y}-\left(\alpha^{+}-\alpha^{-}\right) \rho a u_{\perp}}{\rho a^{2}\left(u^{2}+v^{2}\right)}
\end{aligned}
$$

where $u_{\perp}=u \cos \theta+v \sin \theta$ and $u_{\|}=-u \sin \theta+v \cos \theta$. Thus, the intensity of the steady shear is proportional to the vorticity. As for the intensities of the potential solutions, it is seen that at steady state $\left(\alpha^{+}, \alpha^{-} \rightarrow 0\right)$ the intensity of the first potential pattern is proportional to $\|\nabla p \times \vec{u}\|$, and that of the second potential pattern is proportional to $\nabla p \cdot \vec{u}$, as could be expected.

This model adds a completely new element to the discretization: if the steady state solution can be represented up to truncation error by the steady patterns, the unsteady waves will merely act as a stabilization and the advection scheme will not influence the accuracy. Of course, the basis of steady patterns must be well chosen; in its present form, it seems particularly well suited to the computation of subsonic flows.

The following table summarizes the different wave models used in the computations, where the second column shows the number of "active" waves contributing to the residual, that is the waves for which the product $\alpha^{k} \lambda_{m}^{k}$ is non-zero.

\begin{tabular}{|l|l|}
\hline model & number of waves \\
\hline Roe's model C & 6 waves \\
Roe's model D & 6 waves \\
Rudgyard's streamwise splitting & 6 waves \\
Parpia's model & 5 waves \\
Rudgyard's Mach angle splitting & 5 waves $(M>1)$ \\
Characteristic model & 4 waves \\
Roe's steady pattern model & 3 waves $(M<1)$ \\
\hline
\end{tabular}

\section{5 . The problem of entropy generation}

In this study, the performance of the schemes and models are studied in the case of a smooth subsonic solution of the Euler equations, the isentropic flow inside a "sine" shaped channel. The equation for the lower wall is:

$$
y_{\text {wall }}(x)= \begin{cases}0.1 \times(1-\cos (x-1) \pi) & \text { if } 1 \leq x \leq 3 \\ 0 & \text { else. }\end{cases}
$$

The inlet Mach number is $M_{\infty}=0.5$. The exact solution being isentropic, the generation of entropy along the walls is of particular interest, since it affects the accuracy of the numerical solution. In the following, both the effect of the advection scheme and that of the wave model are assessed. The isolines of the entropy $\Sigma$,

$$
\Sigma=\frac{p / p_{\infty}}{\left(\rho / \rho_{\infty}\right)^{\gamma}}-1
$$

are plotted, with an increment $\Delta \Sigma=0.001$.

\subsection{Comparison between advection schemes}

A comparison is made for a given model and the different advection schemes N, LDA and PSI. Results for the 4-wave model are shown in fig 6 . They confirm that the highest accuracy is obtained for the PSI and LDA scheme. Results with other models are less conclusive, suggesting that the property of linearity preservation (PSI and LDA) is lost when the residual is split in more than 4 waves: a zero residual can be split in non-zero contributions, cancelling one another.

Remark the expected non-monotonic behaviour for the LDA solution in fig $6 \mathrm{c}$.

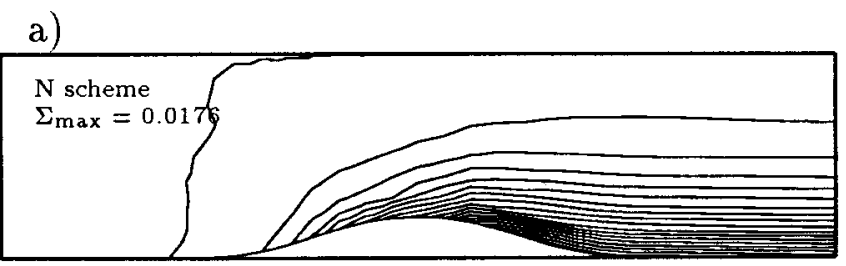

b)

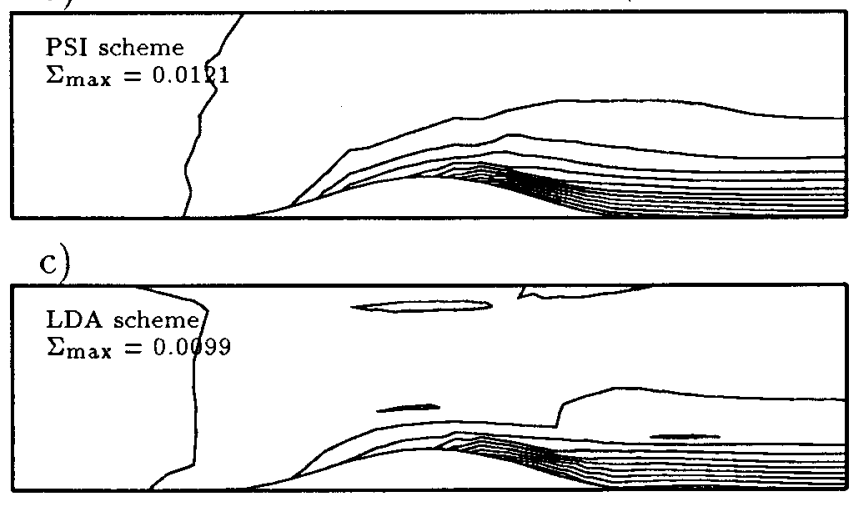

Figure 6: entropy generation for different schemes and the 4-wave model: a) N scheme b) PSI scheme c) LDA scheme

\subsection{Comparison between wave models}

A comparison between the different wave models is now made, to show the problem of entropy generation. A coarse grid of $33 \times 9$ nodes is chosen, and the $\mathrm{N}$ scheme is used to convect the scalar fluctuations. Fig 7 shows the entropy contours obtained with the different models: the streamwise splitting, models $C$ and D, Parpia's model, the characteristic model and the steady pattern model. Clearly, the latter produces the best solution, whereas the 6 -wave models are much less accurate.

\subsection{Two model problems}

- In [4] (pp. 12-14), van Leer explained how the misinterpretation of the flow at a stagnation region by Roe's 
a)
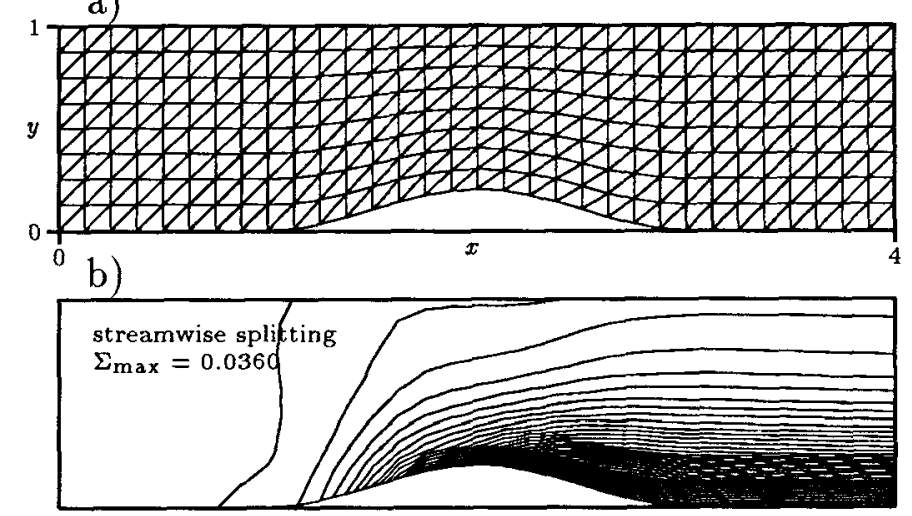

c)

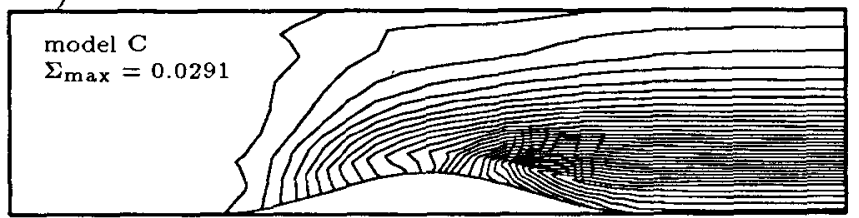

d)

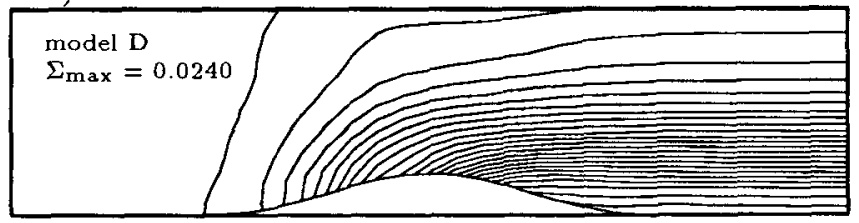

e)

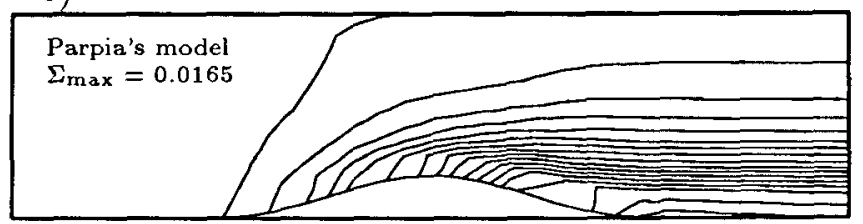

f)

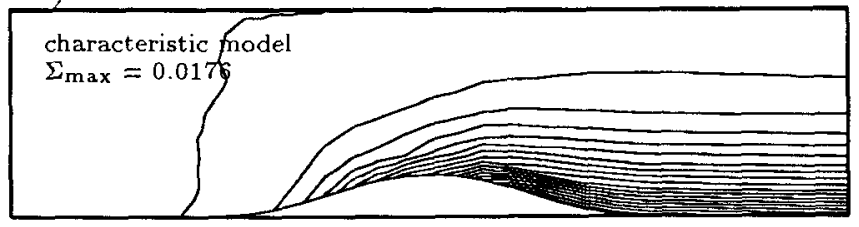

g)

steady pattern model

$\Sigma_{\max }=0.0066$

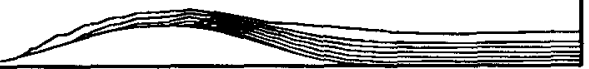

Figure 7: entropy generation for different wave models and the $N$ scheme: a) grid $33 \times 9$, b) streamwise splitting, c) model C, d) model D, e) Parpia's model, f) characteristic model and g) steady pattern model grid aligned Riemann solver can result in the production of entropy. A similar explanation can be given here for the multidimensional wave models:

Consider the simplified model problem consisting of a flow pattern with arbitrary linear variations of the velocity (with the condition $u_{x}+v_{y}=0$ ) and small variations of pressure or density. This pattern, characterized by three degrees of freedom $\left(u_{x}, u_{y}\right.$ and $\left.v_{x}\right)$, can be represented by two shear waves propagating in directions $\theta_{1}$ and $\theta_{2}$ with strengths $\alpha_{1}$ and $\alpha_{2}$ :

$$
\left[\begin{array}{cc}
0 & 0 \\
u_{x} & u_{y} \\
v_{x} & v_{y} \\
0 & 0
\end{array}\right]=\alpha_{1} \mathbf{P}_{u}^{s}\left(\theta_{1}\right)+\alpha_{2} \mathbf{P}_{u}^{s}\left(\theta_{2}\right)
$$

where for instance, $\theta_{2}$ is chosen so as to minimize $\alpha_{1}$ and $\alpha_{2}$. This pattern recognition is "correct" in the sense that individually, each wave brings no change of pressure or density. But the same flow pattern can also be interpreted as due to four orthogonal acoustic waves with strengths $\pm \alpha_{1}$ propagating in direction $\theta_{1}$, and a shear wave propagating in an arbitrary direction $\theta_{2}$, with strength $\alpha_{2}$ :

$$
\begin{aligned}
& {\left[\begin{array}{cc}
0 & 0 \\
u_{x} & u_{y} \\
v_{x} & v_{y} \\
0 & 0
\end{array}\right]=\alpha_{1}\left\{\mathbf{P}_{u}^{a}\left(\theta_{1}\right)-\mathbf{P}_{u}^{a}\left(\theta_{1}+\pi / 2\right)\right.} \\
+\quad & \left.\mathbf{P}_{u}^{a}\left(\theta_{1}+\pi\right)-\mathbf{P}_{u}^{a}\left(\theta_{1}+3 \pi / 2\right)\right\}+\alpha_{2} \mathbf{P}_{u}^{s}\left(\theta_{2}\right)
\end{aligned}
$$

This interpretation of the flow is not correct, and will result in non-zero pressure updates, which, in turn, will generate entropy. To illustrate the problem, the models are tested in a triangular cell representing a stagnation flow. The conditions are $u_{x}=-U / \Delta h, u_{y}=v_{x}=0$, and $v_{y}=U / \Delta h$. Density and pressure variations are neglected. Fig 8 shows the active waves for Roe's 6wave model $\mathrm{D}$, Parpia's 5-wave model and the 4-wave characteristic model. Model D interprets the flow as the superposition of 4 acoustic waves, Parpia's model as that of two shear waves and the characteristic model as the superposition of one shear and two acoustic waves. Consequently, the entropy error with model $\mathrm{D}$ will be larger than with Parpia's model. However, this does not explain why Parpia's model does not do better.

- Another aspect of the problem is illustrated by the following model experiment. A triangular cell is considered, upon which is imposed a steady state solution, consisting of a potential flow turning along the wall, as sketched in fig 9 .

$$
\nabla \mathbf{V}=\beta^{p o t 1} \mathbf{P}_{s}^{p o t 1}+\beta^{p o t 2} \mathbf{P}_{s}^{p o t 2}
$$

For a given "average" state, $\beta^{\text {pot } 1}$ and $\beta^{\text {pot2 }}$ can be computed from the conditions that $v_{\text {node }} 1=0$ and $v_{\text {node } 2} / u_{\text {node } 2}=\tan \delta$, where $\delta$ represents the flow angle at node 2. This is a steady solution of the Euler 

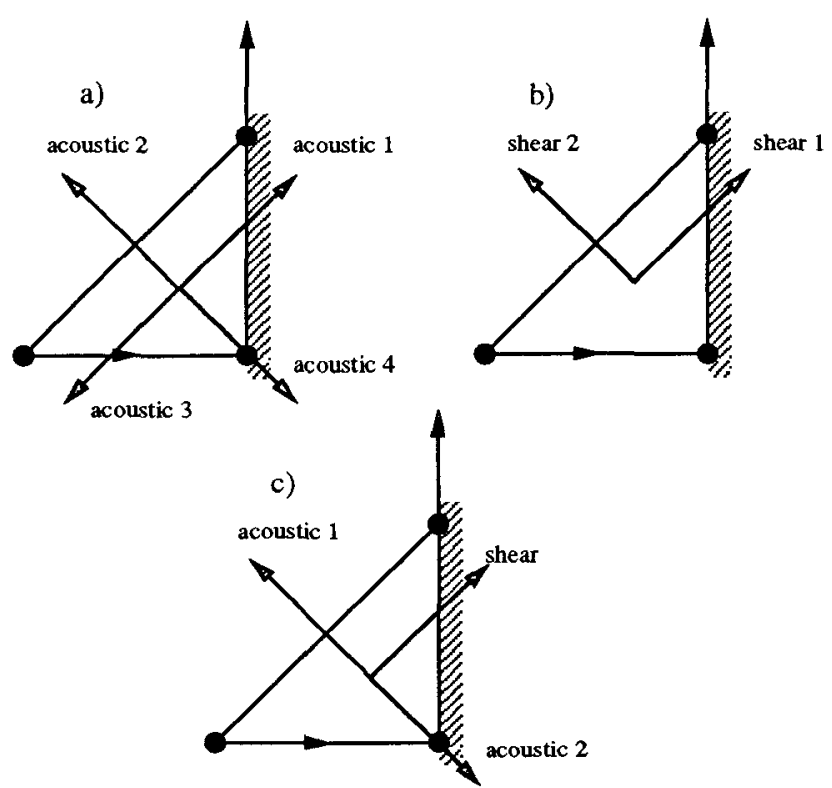

Figure 8: different interpretations of a flow pattern: a) model D, b) Parpia's model, c) characteristic model

equations, therefore the flux balance is zero. Now, a model will preserve this solution if:

$$
\forall \text { wave, } \quad \nabla W^{k} \cdot \widehat{\overrightarrow{\lambda^{k}}}=0,
$$

meaning that each scalar residual is identically zero. On the contrary, if the waves are non-zero and cancel one another, even a linearity-preserving scheme cannot preserve the solution. In fig 10 , the pressure updates $\Delta p_{i}$ to the three nodes are plotted versus the angle $\delta$. It is shown that the 4 -wave model as well as the 3 -wave model (by construction) preserve the solution. On the other hand, model $\mathrm{D}$, the streamwise splitting and Parpia's model destroy it. It is to be noted that the entropy contours shown in fig 7 are consistent with the pressure updates: the largest $\Delta p_{i}$ are indeed associated with the largest entropy generation.

\section{An accuracy study}

An accuracy study is made for a given wave-model and different advection schemes. A smooth continuous flow is computed on a series of grids of decreasing mesh size, and the "error" estimated. The flow chosen is a shear layer at a constant angle with the grid $\left(30^{\circ}\right)$. Taking $z=y-\tan \left(30^{\circ}\right) x$, the density profile chosen is given by:

$$
\rho(z)= \begin{cases}1.0 & \text { if } z<0 \\ 1.0 & \text { if } z>1 \\ 1.0-0.5 \sin ^{2}(\pi z) z(z-1) & \text { else. }\end{cases}
$$

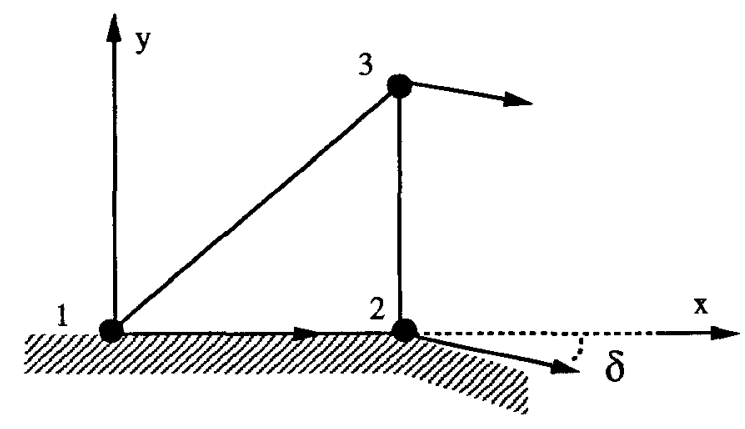

Figure 9: Potential flow turning

and the velocity profile given by:

$$
V(z)= \begin{cases}500 & \text { if } z<0 \\ 550 & \text { if } z>1 \\ 500+50\left(6 z^{5}-15 z^{4}+10 z^{3}\right) & \text { else. }\end{cases}
$$

with $u=V \cos \left(30^{\circ}\right), v=V \sin \left(30^{\circ}\right)$ and $p=10^{5}$. The error between the numerical solution and the exact solution is taken as:

$$
\|\epsilon\|_{L_{2}}=\sqrt{\frac{\sum_{i}^{N}\left[\rho_{\text {num }}\left(x_{i}, y_{i}\right)-\rho_{\text {exact }}\left(x_{i}, y_{i}\right)\right]^{2}}{N}}
$$

The study was made using the N, LDA and PSI schemes for the convection part, and Roe's model $\mathrm{D}$ for the wave modeling part. The domain $(x, y) \in[0,1] \times[-1,2]$ was triangulated into a series of isotropic grids. The error is plotted in a logarithmic graph $\log \|\epsilon\|_{L_{2}}=f(\log \Delta h)$, see fig 11. The slope of the curve determines the order of accuracy of the scheme, giving 0.65 for the $\mathrm{N}$ scheme, 1.60 for the PSI scheme and 2.2 for the LDA scheme. These values agree well with those obtained in the scalar case [9].

This accuracy study is certainly not complete, since one could argue that only two unknowns, namely $V$ and $\rho$, actually vary. A study where all the unknowns undergo changes has to be conducted to assess the accuracy of the schemes for complex flows. However, it can be concluded from this study that the linear $\mathrm{N}$ scheme is not sufficient to guarantee high accuracy.

\section{Numerical results}

The fluctuation-splitting schemes are now tested on 6 testcases representative of different flow regimes: (1) the supersonic flow over a wedge in a channel, (2) the supersonic and subsonic flows in a sine-shaped channel (3) the subcritical flow over a cylinder, (4) the subcritical flow over a NACA 0012 airfoil, (5) the transonic flow over a NACA 0012 airfoil, and (6) the hypersonic flow over a cylinder. One of the big advantages of the method lies in its natural application to unstructured meshes, 

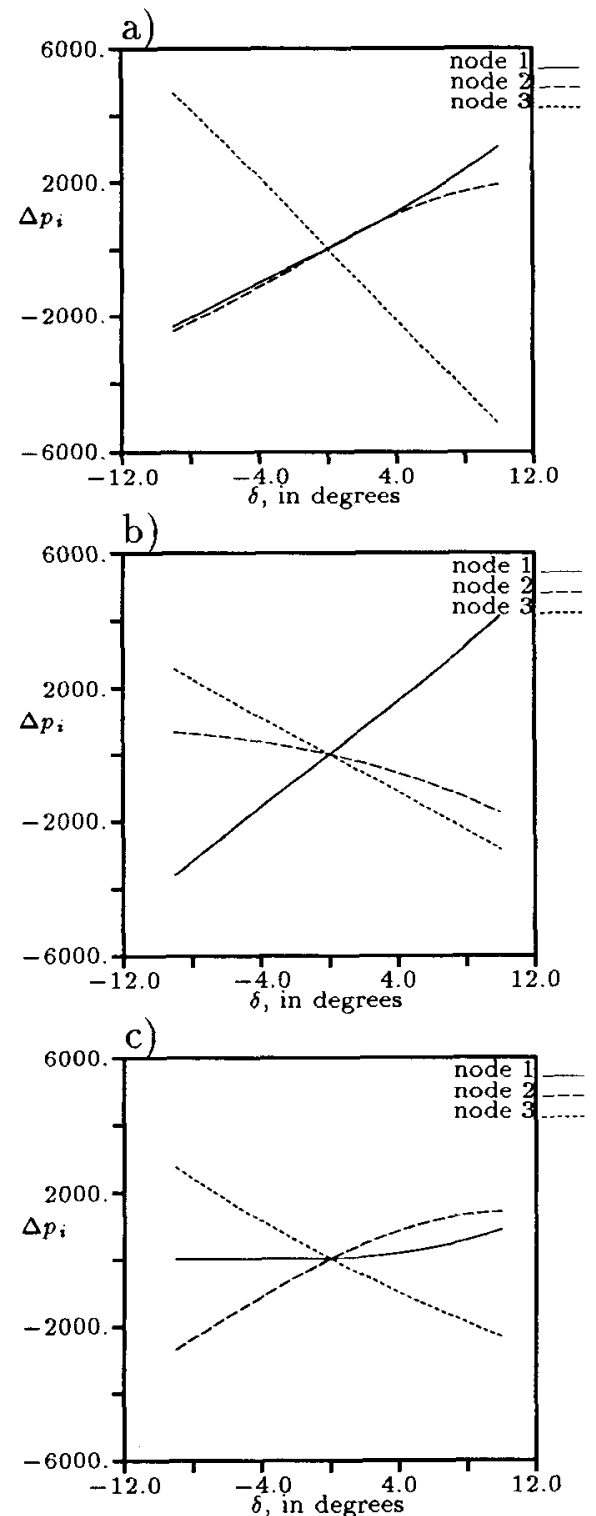

d)

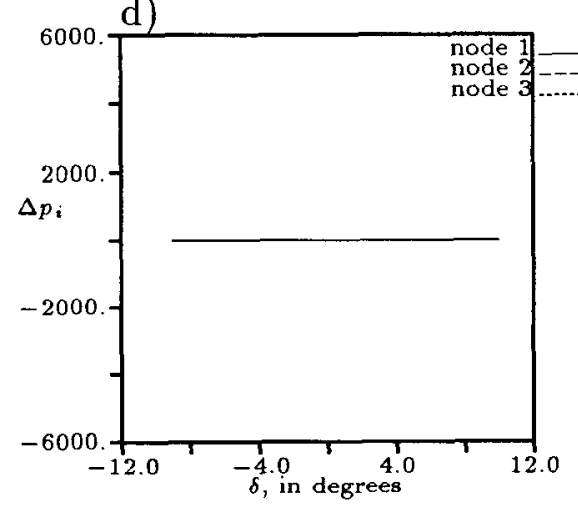

Figure 10: pressure updates: a) streamwise splitting, b) model D, c) Parpia's model, d) characteristic model and steady pattern model

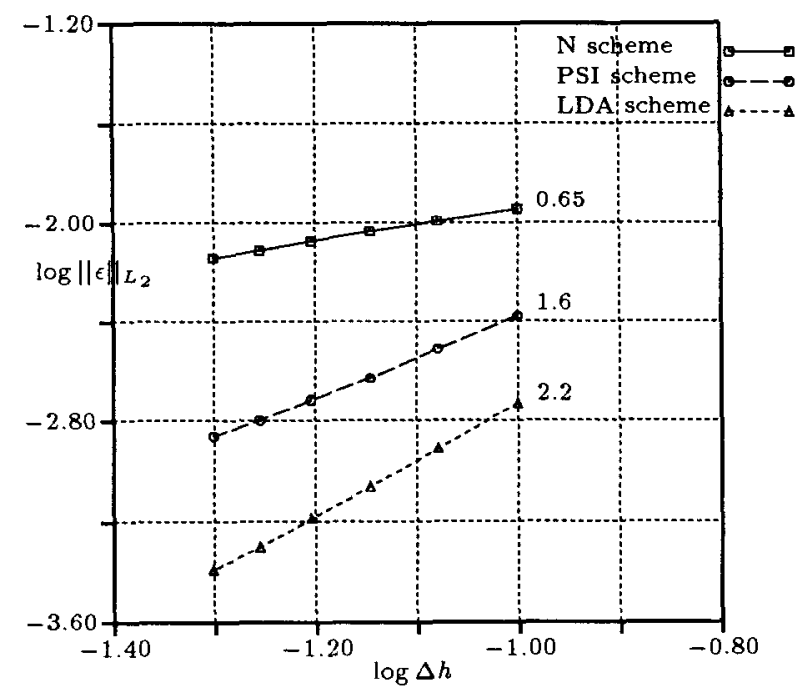

Figure 11: Order of accuracy of the schemes

since all computations are done on a triangle basis. The unstructured meshes used subsequently were generated using a Delaunay frontal method by J.-D. Müller [21].

\section{$7.115^{\circ}$ wedge channel}

The flow in a channel at $M_{\infty}=2.0$ is considered. Due to the presence of a wedge, an oblique shock wave is produced with reflects at the upper wall. At the tip of the wedge, a Prandtl-Meyer fan is produced, which interacts with the reflecting shock. Fig 12 shows the grid and the Mach number contours obtained respectively with model C, Parpia's model, the characteristic model, and the Mach angle splitting, all used with the PSI scheme. The solution obtained with the Mach angle splitting and the $\mathrm{N}$ scheme is also shown: this scheme is more diffusive than the PSI and results in a smearing of the shocks. In all cases, the shock and the expansion fan are well resolved; however, Parpia's model has problems dealing with the regions of interaction between the expansion fan and the reflected shock, as does the characteristic model. The best solution, perhaps not surprisingly, is obtained with the Mach angle splitting and the PSI scheme.

\subsection{Flow in a sine channel}

This problem is the well-known problem of Powell and van Leer [22], where the flow in a symmetric sine channel is computed. Here, the channel is prolongated in the upstream and downstream directions by straight walls. Inflow Mach numbers are $M_{\infty}=1.75$ for the supersonic case and $M_{\infty}=0.5$ for the subsonic case.

Supersonic case: 
a)

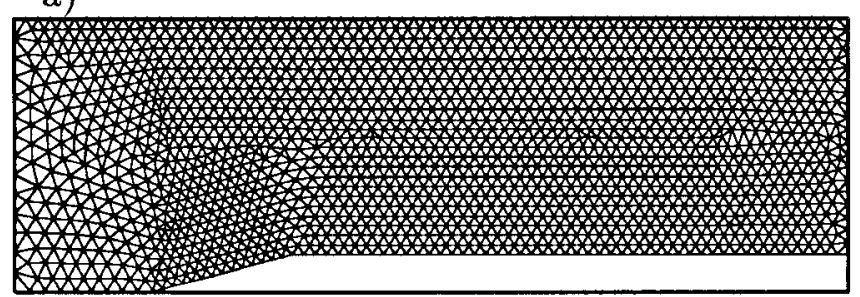

b)

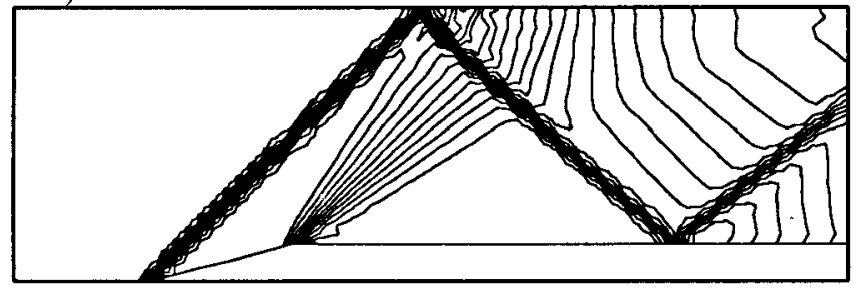

c)

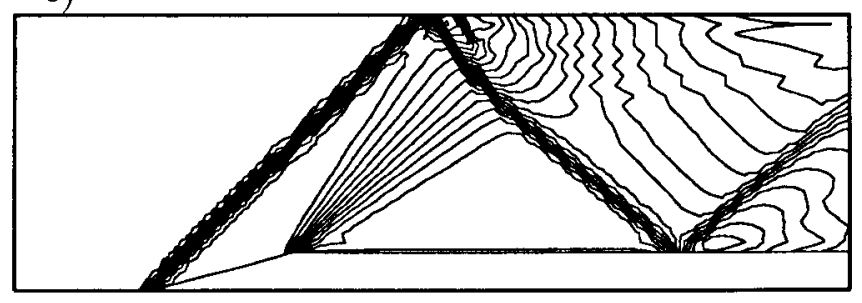

d)

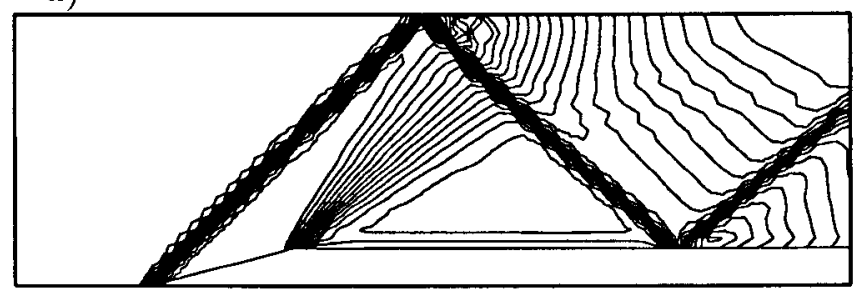

e)

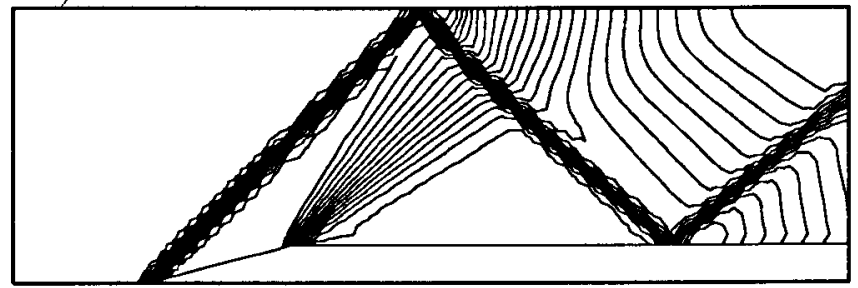

f)

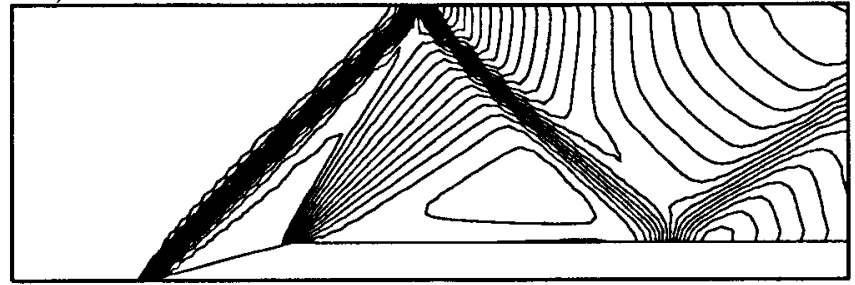

Figure 12: $15^{\circ}$ wedge: a) grid, Mach number contours: b) model C, c) Parpia's model, d) characteristic model, e) Mach angle splitting, f) Mach angle splitting ( $N$ scheme)
Fig 13b shows the Mach number contours obtained with model $\mathrm{C}$ whereas fig $13 \mathrm{c}$ shows the contours obtained with Parpia's model. Both were used in conjunction with the PSI scheme. In the first case, the compression waves coalescing into shock waves, the reflected shocks and the regions behind those shocks are clearly defined. With Parpia's model, the flow is not as well resolved, just as in the wedge case.

Subsonic case:

Fig 13d shows the Mach number contours obtained with the steady pattern model and the $\mathrm{N}$ scheme. The solution is very symmetric and is characterized by very low entropy levels $\left(\Sigma \approx 10^{-4}\right)$, confirming the appropriateness of that model for computing potential flows.

\section{$\underline{7.3 \text { Subcritical flow over a cylinder }}$}

The flow over a cylinder at $M_{\infty}=0.38$ is computed. The mesh, shown in fig 14a, consists of 2900 nodes, with 120 nodes on the body. The theoretical solution is a potential solution (irrotational, isentropic) with no lift and no drag. In a first computation, the steady pattern model is used together with the $\mathrm{N}$ scheme, producing small values of lift and $\operatorname{drag}\left(C_{L}=0.023\right.$ and $\left.C_{D}=-0.007\right)$ and a maximum entropy error $\Sigma_{\max }=$ $5 \times 10^{-3}$ which is very small. The Mach number contours are shown in fig $14 \mathrm{~b}$, confirming the high degree of symmetry of the solution. This solution compares well with the best solutions obtained in the GAMM workshop [23], where this testcase was proposed. In a second computation, model D and the PSI scheme are used, and the results confirm the analysis of section 5: a large entropy error $\Sigma_{\max }=9 \times 10^{-2}$, and consequently an increase in drag $\left(C_{D}=0.880\right)$. The Mach number contours are shown in fig 14c, where the lack of symmetry of the solution is clearly visible. Finally, the Mach number distribution along the body is plotted for both solutions in fig 15.

\subsection{Subcritical NACA0012 Airfoil}

The subcritical flow $\left(M_{\infty}=0.63, \alpha=2^{\circ}\right)$ around a NACA0012 airfoil is computed. The grid consists of 1811 nodes, with 128 nodes on the body, see fig $16 \mathrm{a}$. The far-field boundary is at 20 chords, and a pointvortex correction was applied. For the first computation, model D and the PSI schemes were used. The scheme converged to machine zero, with values of lift $\left(C_{L}=0.25\right)$ and $\operatorname{drag}\left(C_{D}=0.042\right)$ which are far from the correct values [23] $\left(C_{L} \approx 0.33\right.$ and $\left.C_{D}=0.0\right)$. This can be attibuted to the entropy layer generated at the leading edge of the airfoil $\left(\Sigma_{\max }=0.12\right)$. For the second computation, the steady pattern model was used together with the $\mathrm{N}$ scheme. This time, the residual only dropped 2 orders of magnitude, but the lift and drag stabilized around the values $C_{L}=0.33$ and $C_{D}=0.001$ which is quite accurate. About 12 times less entropy 
a)

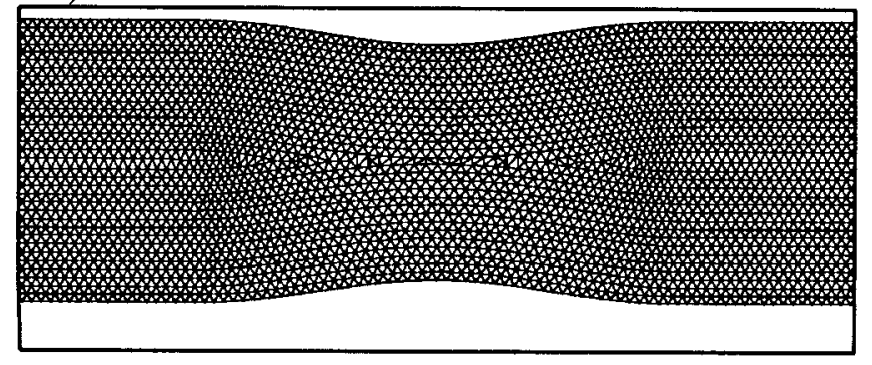

b)

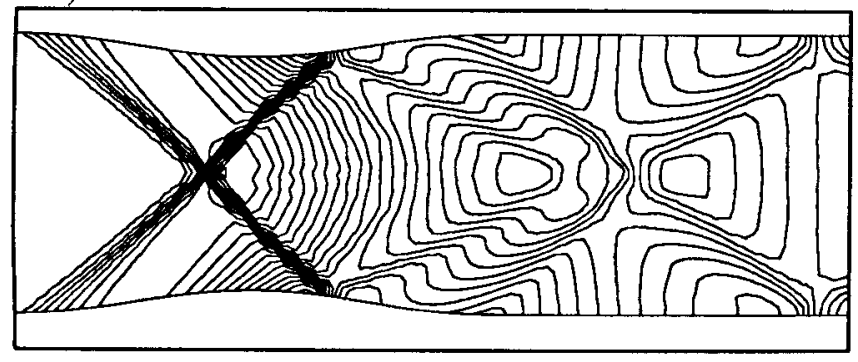

c)

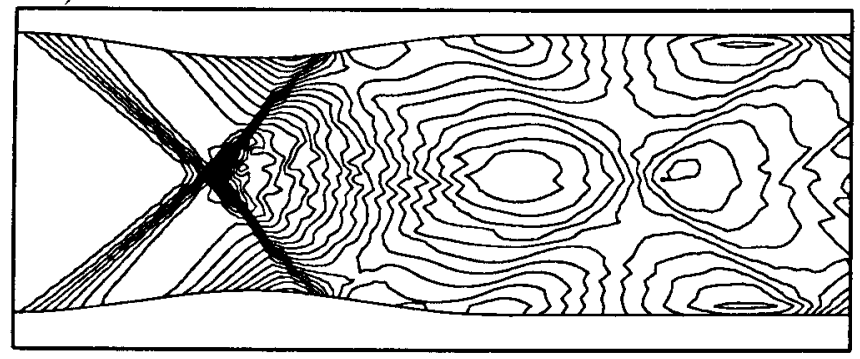

d)

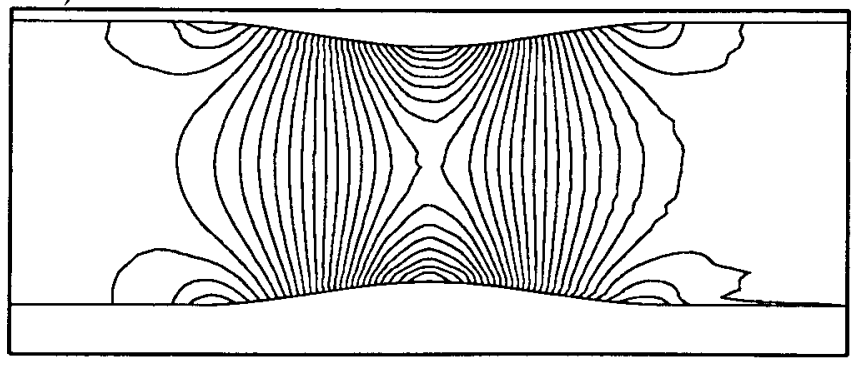

Figure 13: a) detail of the grid, b) Mach number contours $\left(M_{\infty}=1.75\right)$ model C, c) Mach number contours $\left(M_{\infty}=1.75\right)$ Parpia's model, d) Mach number contours $\left(M_{\infty}=0.5\right)$ steady pattern model

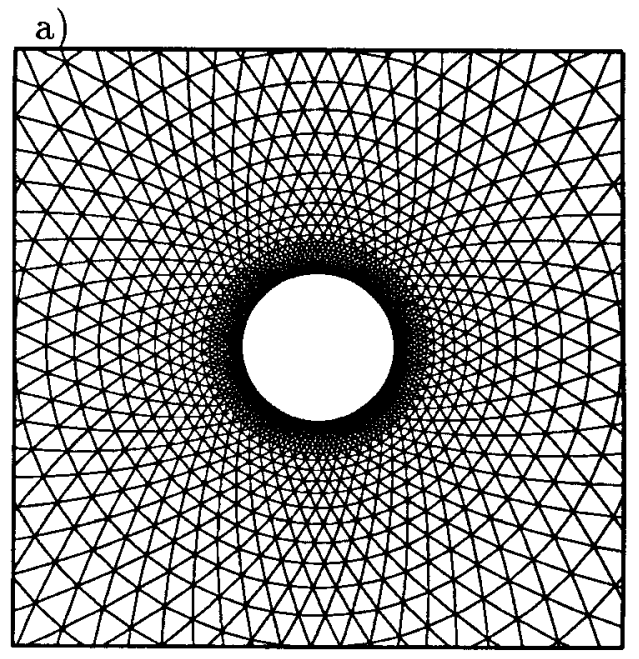

b)

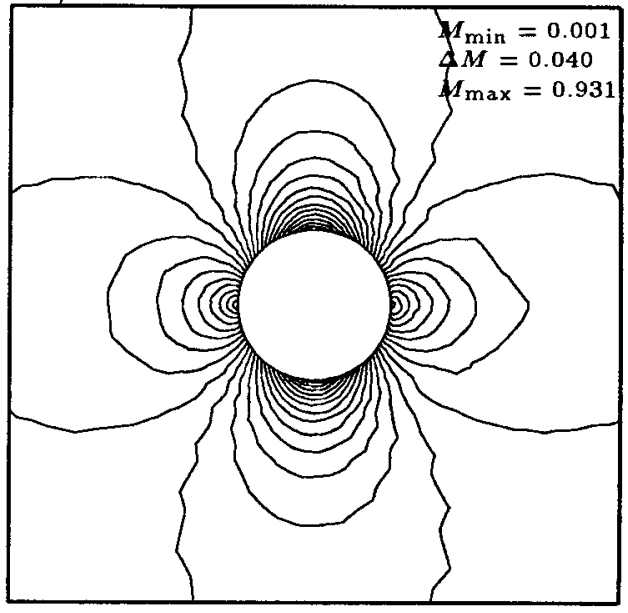

c)

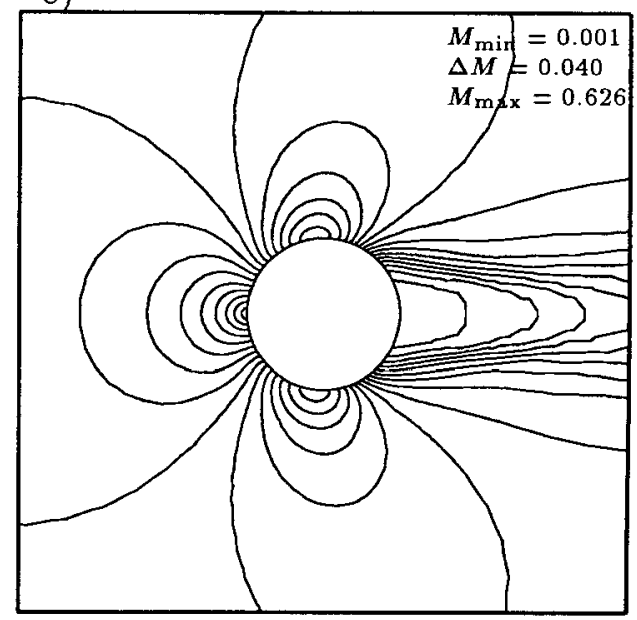

Figure 14: cylinder $M_{\infty}=0.38:$ a) grid, b) Mach number, steady pattern model and N scheme, c) Mach number, model D and PSI scheme 


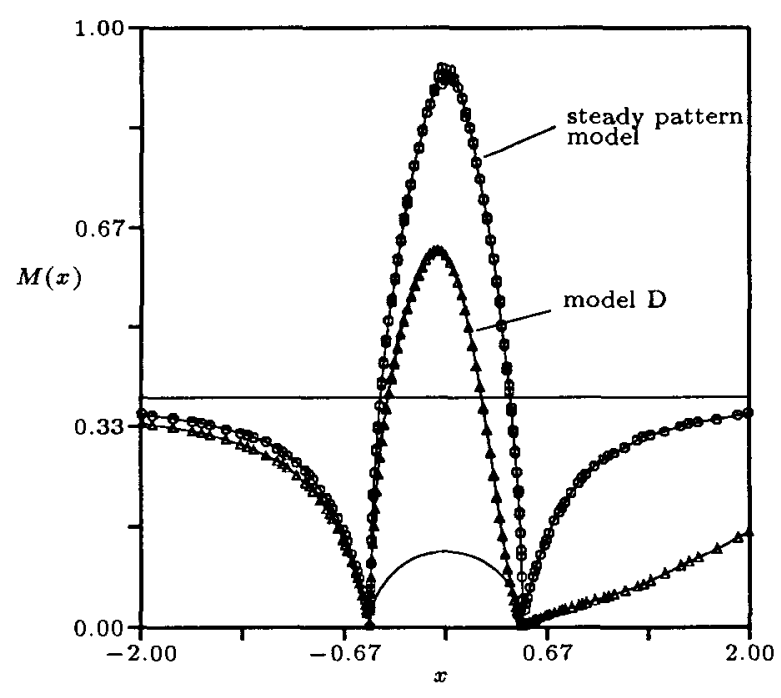

Figure 15: cylinder $M_{\infty}=0.38$ : Mach number along the body

is generated with this model $\left(\Sigma_{\max }=0.01\right)$ than with model D. The Mach number profiles for the two computations are compared in fig $16 \mathrm{~b}$. The pressure coefficient for the steady pattern model solution is plotted in fig $16 \mathrm{c}$.

\subsection{Transonic NACA0012 Airfoil}

The critical flow $\left(M_{\infty}=0.85, \alpha=1^{\circ}\right)$ over a NACA0012 airfoil is computed. A blending of two models is used in this computation: to avoid excessive entropy generation near the leading edge, the steady pattern model is used in the subsonic region of the flow, whereas the 4-wave model is used elsewhere. The grid used contains 9000 cells, 4600 nodes, with 128 nodes on the airfoil. The far-field boundary is at 20 chords and a point-vortex correction is applied. The values of lift and drag $C_{L}=0.354$ and $C_{D}=0.057$ agree well with those of reference solutions. Fig 17a shows the Mach number contours for this solution, fig $17 \mathrm{~b}$ the Mach number profile on the body, and fig $17 \mathrm{c}$ the entropy profile on the body.

\subsection{Hypersonic flow over a cylinder}

The flow at Mach 8 over a cylinder is computed. A grid similar to that shown in fig 14 is used. The flow is characterized by a strong bow shock in the front part, and a more complex structure in the low density region at the rear part of the cylinder, with shocks and separations. Some reference solutions for this severe testcase can be found in [23].

Careful choice of the CFL number is needed to pre- a)

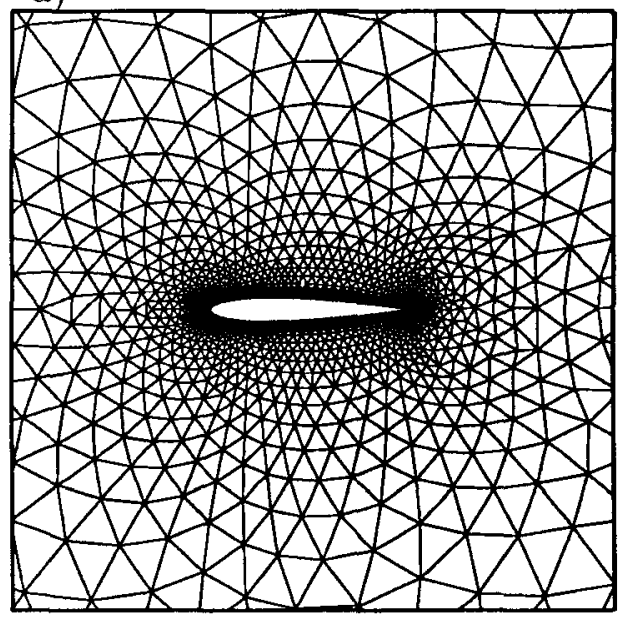

b)

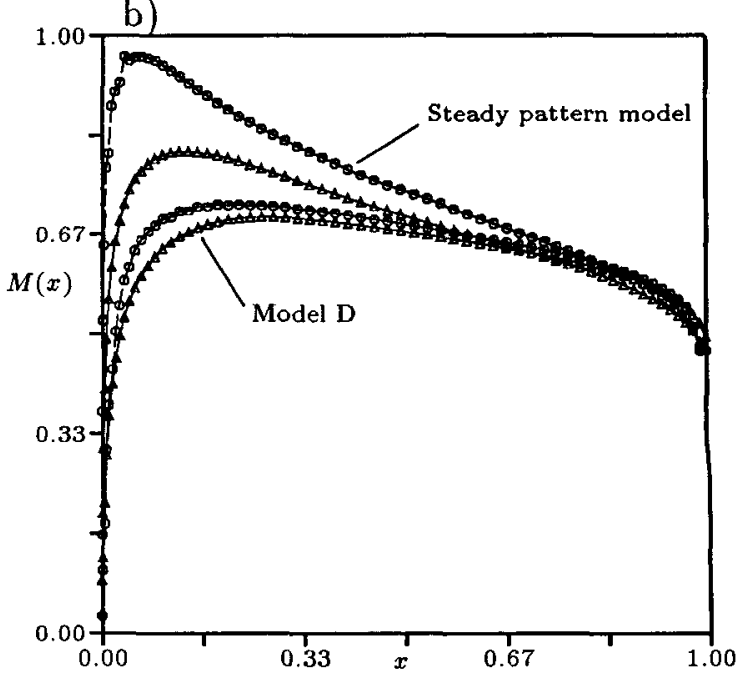

c)

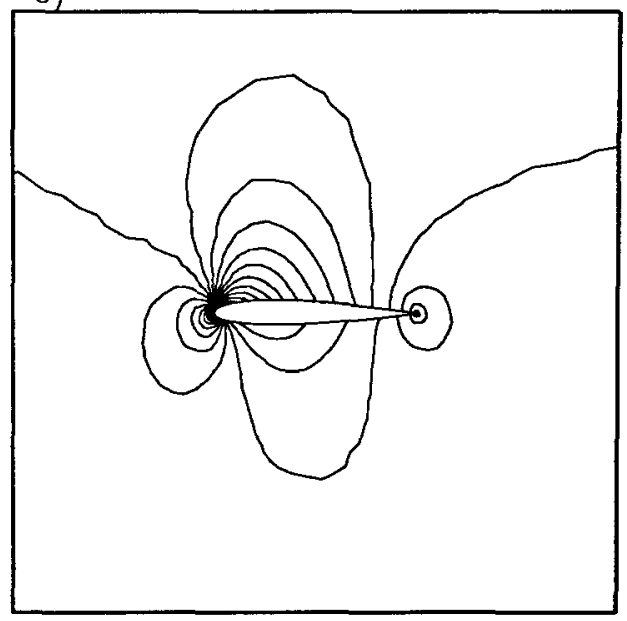

Figure 16: NACA0012 $M_{\infty}=0.63, \alpha=2^{\circ}$ : a) grid, b) Mach number profiles along the airfoil for model $D$ and the steady pattern model, c) pressure coefficient for the steady pattern model 
a)
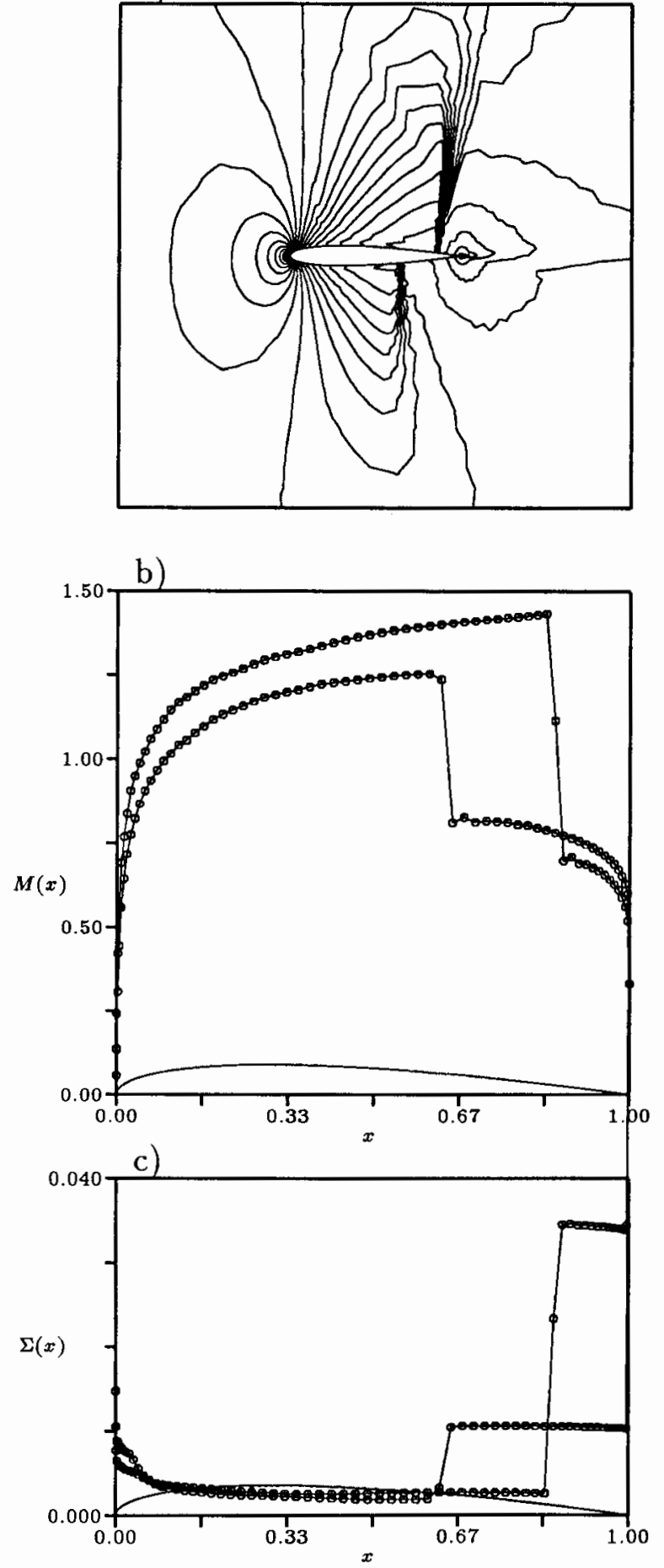

Figure 17: NACA0012 $M_{\infty}=0.85, \alpha=1^{\circ}$ : steady pattern model + characteristic model and PSI scheme a) Mach number, b) Mach number profile, c) entropy profile vent the appearance of negative pressures during the transient phase. In the following, the results obtained using the $\mathrm{N}$ scheme and the Mach angle splitting are shown, since the combination proved a relatively stable one. Since the Mach angles are not defined in subsonic flows, an empirical fix [17] is used, whereby $\sqrt{M^{2}-1}$ is replaced by $\sqrt{\max \left(\left|M^{2}-1\right|, \epsilon\right)}$. In fig 18a, the Mach number contours are shown. A detailed view of the velocity field in the rear part of the cylinder is shown in fig 18b: the recirculation is clearly visible, but more points are needed there for a better resolution of the flow.
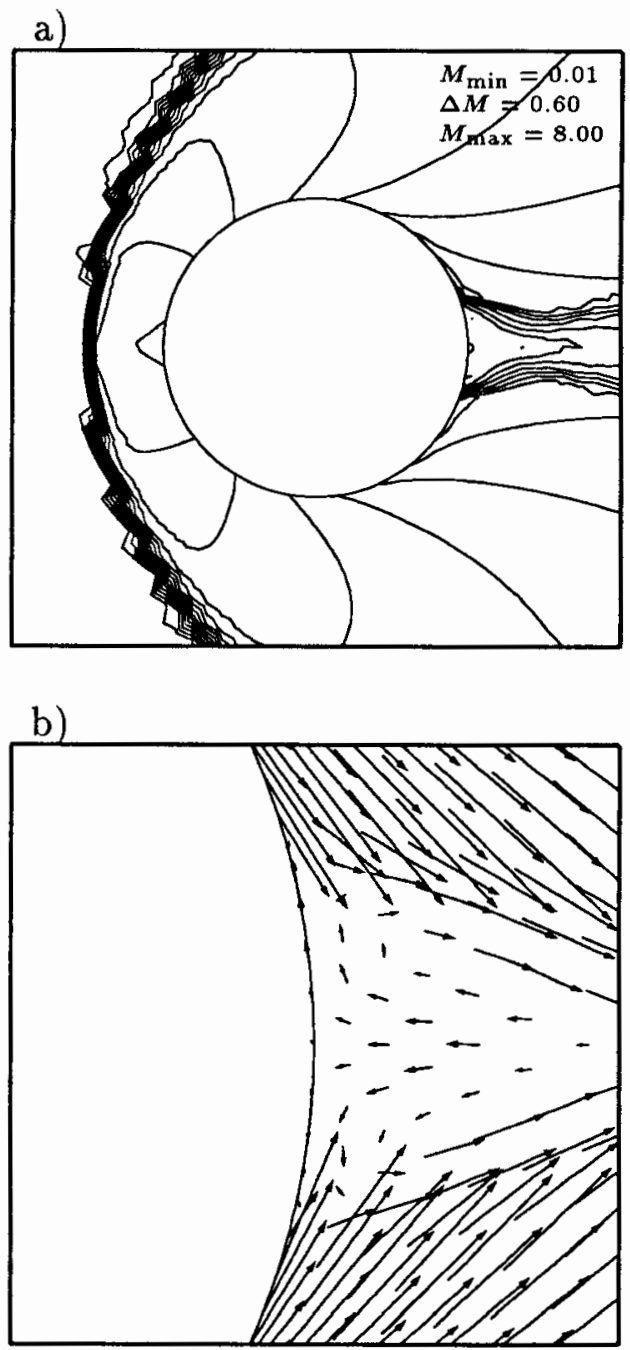

Figure 18: cylinder $M_{\infty}=8.0$, a) Mach number, b) detailed view of the velocity field

\section{Conclusions}

A detailed study is made of the wave modeling step in the multidimensional upwind fluctuation-splitting Euler solvers. While most models perform well for supersonic 
flows with shocks, it is found that wave models with 5 or more active waves lead to excessive false entropy generation along curved walls in subsonic flow. The new model combining steady patterns and three active simple waves performs extremely well in this respect, but fails in presence of shocks. An optimal compromise at present seems to lie in a combination of the 3 and 4wave models as a function of the flow regime.

The accuracy of the different advection schemes has been evaluated numerically for the Euler solver in the case of a shear flow, confirming the accuracy of 0.85 for the linear monotone $N$ scheme, 2.0 for the linear non-monotone LDA scheme and 1.6 for the non-linear monotone PSI scheme obtained previously for scalar advection equation.

As a general conclusion, the paper stresses that wavemodelling, although improved in recent times, is still a topic of further research.

\section{Acknowledgements}

The research at VKI was supported by the Commission of the European Communities through research contract AER2-CT92-0040/PL-2037, within the Brite/Euram programme, monitored by Dr. Borthwick. The authors would also like to thank Dr. M. Rudgyard (Cerfacs, France) for helpful discussions on wavemodelling and multidimensional upwinding.

\section{References}

[1] S.F. Davis. A rotationally biased upwind difference scheme for the Euler equations. J. Comp. Physics, Vol. 56, pp. 65-92, 1984.

[2] C.L. Rumsey, B. van Leer, and P.L. Roe. A Multidimensional Flux Function with Applications to the Euler and Navier-Stokes Equations. J. Comp. Physics, Vol. 105, pp. 306-323, 1993.

[3] P.L. Roe. Beyond the Riemann problem I. ICASE$L A R C$ Workshop on 'Alg. trends for the 90s', Hampton, $V A$, September, 1991, to be published by Springer, 1992.

[4] B. van Leer. Progress in multi-dimensional differencing. ICASE Report No. 92-43, 1992.

[5] P.L. Roe. Linear advection schemes on triangular meshes. CoA Report No. 8720, Cranfield Institute of Technology, November, 1987.

[6] R. Struijs, H. Deconinck, and P.L. Roe. Fluctuation Splitting Schemes for multidimensional convection problems : an alternative to finite volume and finite element methods. VKI LS 1990-03, Computational Fluid Dynamics, 1990.

[7] G. Bourgois, H. Deconinck, and P.L. Roe. Multidimensional upwind schemes for scalar advection on tetrahedral meshes. Proc. 1st European CFD Conf., Brussels, Vol. 1, Elsevier, September, 1992.
[8] H. Deconinck, H. Paillère, R. Struijs, and P.L. Roe. Multidimensional upwind schemes based on fluctuation-splitting for systems of conservation laws. J. Comp. Mechanics, to appear, 1993.

[9] H. Deconinck, R. Struijs, G. Bourgois, and P.L. Roe. Compact advection schemes on unstructured grids. VKI LS 93-04, Computational Fluid Dynamics, March, 1993.

[10] P.L. Roe, R. Struijs, and H. Deconinck. A conservative linearization of the multidimensional Euler equations. to appear, J. Comp. Phys., 1993.

[11] R. Struijs, H. Deconinck, and P.L. Roe. Fluctuation Splitting Schemes for the 2D Euler Equations. VKI LS 1991-01, Computational Fluid Dynamics, 1991.

[12] P.L. Roe. Discrete Models for the numerical analysis of time-dependent multidimensional gas dynamics. Journal of Computational Physics, Vol. 63, 1986.

[13] H. Deconinck, Ch. Hirsch, and J. Peuteman. Characteristic decomposition methods for the multidimensional Euler equations. Lecture Notes in Physics, vol. 264, Springer, 1986.

[14] P. De Palma, H. Deconinck, and R. Struijs. Investigation of Roe's 2D Wave Decomposition Models for the Euler Equations. VKI Technical Note 172, July, 1990.

[15] P.L. Roe and L. Beard. An improved wave model for multidimensional upwinding of the Euler equations. 13th Int. Conf. on Numerical Methods in Fluid Dynamics, Rome, July, 1992.

[16] M. Rudgyard. Multidimensional Wave Decompositions for the Euler Equations. VKI LS 93-04, Computational Fluid Dynamics, March, 93.

[17] M. Rudgyard. A comparison of multidimensional upwinding for cell-vertex schemes on triangular meshes. Proc. Conf. on Numerical Methods for Fluid Dynamics, Reading, Oxford University Press, April, 1992.

[18] I.H. Parpia and D.J. Michalek. A nearly-monotone genuinely multi-dimensional scheme for the Euler equations. AIAA-92-035, 1992.

[19] P.L. Roe. Wave-modelling of time-dependent hyperbolic systems. ICASE Report, to be published, 1993.

[20] R. Struijs, H. Deconinck, P. De Palma, P.L. Roe, and K.G. Powell. Progress on Multidimensional Upwind Euler Solvers for Unstructured Grids. AIAA-91-1550, June, 1991.

[21] J.-D. Müller, P.L. Roe, and H. Deconinck. Delaunaybased triangulations for the Navier-Stokes equations with minimum user input. 13th Int. Conf. on Numerical Methods in Fluid Dynamics, Rome, June, 1992.

[22] K.G. Powell and B. van Leer. A genuinely multidimensional upwind cell-vertex scheme for the Euler equations. AIAA-89-0095, 1989.

[23] A. Dervieux, B. van Leer, J. Periaux, and A. Rizzi (Eds.). Numerical Simulation of Compressible Euler Flows. Notes on Numerical Fluid Mechanics, Vol. 26, Vieweg, 1989. 